\title{
Requerimiento de proteína y lípidos para el crecimiento de juveniles del pez nativo Dormitator latifrons (Richardson, 1844)
}

\section{Protein and lipid requirements for growth of juveniles of the native fish Dormitator latifrons (Richardson, 1844)}

\author{
Daniel Badillo-Zapata ${ }^{1,2 *}$, Fredy De Jesús Zaragoza ${ }^{3}$, Fernando Vega-Villasante ${ }^{1}$, Jorge Manuel \\ López-Huerta $^{1}$, Selene Herrera-Resendiz ${ }^{1}$, Leyver Cueto-Cortés ${ }^{1}$, Saul Rogelio Guerrero-Galvan ${ }^{1}$ \\ ${ }^{1}$ Universidad de Guadalajara, Centro Universitario de la Costa, Av. Universidad 203, delegación Ixtapa, CP. 48280, Puerto \\ Vallarta, Jalisco, México. \\ ${ }^{2}$ Cátedras CONACYT. Consejo Nacional de Ciencia y Tecnología. México \\ ${ }^{3}$ Instituto Tecnológico Superior de Tlatlauquitepec, Tlatlauquitepec, Puebla, México. \\ *Autor de correspondencia: danielbad00@hotmail.com \\ Nota científica recibida: 13 de julio de 2017 aceptada: 01 de febrero de 2018
}

RESUMEN. Dormitator latifrons es un pez con potencial comercial que habita ambientes con climas tropicales y subtropicales desde el sur de California (EEUU) hasta Perú. El objetivo fue determinar los requerimientos nutricionales de proteína y lípidos para el crecimiento de juveniles, se elaboraron cuatro dietas experimentales con contenidos de proteína del 30 y $40 \%$, y lípidos del 8 y 16\%, con tres repeticiones por tratamiento. Se realizó el análisis proximal de las dietas experimentales, del músculo al inicio y al final del experimento y se determinó la digestibilidad aparente. No se detectaron diferencias significativas $(p>0.05)$ en el crecimiento, supervivencia y la composición proximal del músculo de los peces. La digestibilidad aparente de la dieta con $30 \%$ de proteína y $8 \%$ de lípidos fue significativamente superior al resto de las dietas, por lo que se considera suficiente para un adecuado crecimiento de juveniles de $D$. latifrons.

Palabras clave: $D$. latifrons, lípidos, nativo, nutrición, proteína

ABSTRACT. Dormitator latifrons is a fish with commercial potential that inhabits environments with tropical and subtropical climates from southern California (USA) to Peru. The objective was to determine the nutritional requirements of protein and lipids for the growth of juveniles; four experimental diets were prepared with protein contents of 30 and $40 \%$, and lipids of 8 and $16 \%$, with three repetitions per treatment. Proximal analysis of the experimental diets, of the muscle at the beginning and at the end of the experiment, was carried out and the apparent digestibility was determined. No significant differences $(p>0.05)$ were detected in the growth, survival and proximal composition of the fish muscle. The apparent digestibility of the diet with $30 \%$ protein and $8 \%$ lipids was significantly higher than the rest of the diets, so it is considered sufficient for adequate growth of $D$. latifrons juveniles.

Key words: $D$. latifrons, lipids, native, nutrition, protein

\section{INTRODUCCIÓN}

En las últimas décadas se ha presentado un crecimiento acelerado de la acuicultura, debido a que se reconoce como actividad productora de proteína de origen animal (Tacon et al. 2011). La FAO reporta que hay alrededor de 580 especies acuícolas cultivadas de forma controlada a nivel mundial. Entre ellas se encuentran 362 peces de escama, 104 moluscos, 62 crustáceos, seis ranas y reptiles, nueve invertebrados acuáticos y 37 plantas acuáticas. La gran mayoría de las especies utilizadas con fines de acuicultura son exóticas o introducidas, que presentan facilidad en su manejo, adaptabilidad y buenos índices de crecimiento (FAO 2016). Pero no se contempla que estas especies pueden ocasionar alteraciones en los ecosistemas, y provocar desplazamiento de especies nativas (Okolodkov et al. 2007). 
En México se realizan estudios para determinar los requerimientos nutricionales óptimos de distintas especies nativas, para desarrollar su potencial de cultivo, entre estas se encuentran el abulón rojo (Haliotis rufescens), atún rojo del Atlántico, (Thunnus thynnus), ostra de Cortéz (Crassostrea corteziensis), totoaba (Totoaba macdonaldi), lenguado de California (Paralichthys californicus), lenguado del mar de Cortéz (Paralichthys aestuarius) y la corvina del Golfo (Cynoscion othonopterus), todos de origen marino (GonzálezFélix et al. 2014, Pérez-Velázquez et al. 2015). Pero el estudio de la nutrición de peces nativos es relativamente nuevo en términos del conocimiento acumulado con otras especies. Actualmente parte de los estudios nutricionales en peces nativos se dirigen a la evaluación de la proteína y los lípidos contenidos en la dieta, y su relación con el crecimiento, reproducción y mantenimiento, para mejorar la producción económica (González-Félix et al. 2014).

El dormilon gordo del Pacífico, chopopo, chame, puyeque o popoyote (Dormitator latifrons Richardson, 1844) es un pez originario de climas tropicales y subtropicales, con preferencia por aguas con temperaturas entre 21 y $30^{\circ} \mathrm{C}$, tiene la capacidad de tolerar aguas salobres y concentraciones de oxígeno bajas de hasta $0.4 \mathrm{mg} \mathrm{L}^{-1}$ (Castro-Rivera et al. 2005, López-López et al. 2015). Su distribución abarca desde el sur de California (EUA) hasta Perú, siendo las ciénegas uno de sus principales hábitats (FAO 2010). Este pez tiene importancia comercial en la región sur y sureste de México, principalmente en las costas de Guerrero y Oaxaca donde se utiliza para consumo humano, mientras que en el centro del país se comercializa su filete (Larumbe 2002). En Ecuador es de gran importancia económica para las comunidades costeras, donde se cultiva de forma artesanal para consumo y exportación (Freire-Lascano 2013). Debido a la falta de información sobre los requerimientos nutricionales de esta especie, el objetivo fue determinar los requerimientos de proteína y lípidos de juveniles de $D$. latifrons mantenidos bajo condiciones controladas.

\section{MATERIALES Y MÉTODOS}

El estudio se realizó en la Universidad de Guadalajara en el Centro Universitario de la Costa en las instalaciones del laboratorio de Calidad de Agua y Acuicultura Experimental en Puerto Vallarta, Jalisco, México. Para lo que se colectaron 200 juveniles de $D$. latifrons, de la laguna el Quelele en Nayarit, México. Los peces se trasladaron al laboratorio en donde se pusieron en tratamiento químico preventivo con triclorfón (Neguvon, Bayer ${ }^{\circledR}$ ) a concentración de $0.3 \mathrm{ppm}$, para eliminar la posible presencia de parásitos internos y externos (VegaVillasante et al. 2017). Los peces se mantuvieron en cuarentena por $15 \mathrm{~d}$, en los que se suministró alimento formulado con $30 \%$ de proteína y $8 \%$ de lípidos (Purina ${ }^{\circledR}$ ), a las 9:00 y 17:00 h.

Se elaboraron cuatro dietas experimentales a base de harina y aceite de pescado con 30 y $40 \%$ de proteína cruda y, 8 y 16\% de lípidos, respectivamente (Tabla 1 ). Los insumos se pesaron en una báscula analítica (Nimbus $\left.{ }^{\circledR}\right)$, con presición de $\pm 0.1 \mathrm{~g}$, posteriormente se mezclaron con una maquina mezcladora (Blazer ${ }^{\circledR}$, modelo 810 ), para tener una pasta homogénea, luego la pasta se pasó a un molino de carne (Torrey ${ }^{\circledR}$ modelo M-12-F5) para obtener pellets de un diámetro aproximado de $3 \mathrm{~mm}$. El material resultante se secó una estufa de convección (NOVATECH ${ }^{\mathrm{R}}$ ) a $80^{\circ} \mathrm{C}$ por $24 \mathrm{~h}$, para luego almacenarlo a $-20{ }^{\circ} \mathrm{C}$, hasta su uso.

El sistema experimental consistió de 12 reservorios de $350 \mathrm{~L}$ de capacidad, conectados con un sistema de recirculación (sistema de biofiltración y lámpara de luz UV), para mantener la calidad del agua. El experimento tuvo una duración de 60 $d$, cada uno de los tratamientos experimentales se realizó por triplicado y se colocaron 10 organismos por unidad experimental. La temperatura se mantuvo a $29.02 \pm 1.0{ }^{\circ} \mathrm{C}$ con ayuda de calentadores con termostato. Se tomaron muestras de agua una vez por semana para determinar la concentración de amoniaco, nitritos, nitratos y $\mathrm{pH}$ (fotómetro YSI $\left.9500^{\circledR}\right)$. El oxígeno disuelto y la temperatura se registró con un oxímetro (YSI $550 \mathrm{~A}^{\circledR}$ ). Al inicio del experimento los peces se alimentaron con una ración 
Tabla 1. Ingredientes, composición próxima, coeficiente de digestibilidad de las cuatro dietas experimentales que contienen diferentes niveles de proteína cruda y grasa cruda utilizada para alimentar a juveniles $D$. latifrons.

\begin{tabular}{lcccc}
\hline & \multicolumn{5}{c}{ Tratamientos experimentales } \\
\cline { 2 - 5 } Ingredientes $\left({\left.\mathrm{g} 100 \mathrm{~g}^{-1}\right)}^{30-8}\right.$ & $30-16$ & $40-8$ & $40-16$ \\
\hline Harina de pescado* & 35.9 & 35.9 & 51.0 & 51.0 \\
Harina de maíz & 5.5 & 5.5 & 5.5 & 5.5 \\
Aceite de pescado & 5.2 & 13.2 & 4.1 & 12.1 \\
Almidón de maíz & 43.7 & 36.0 & 30.0 & 22.0 \\
Gelatina & 6.0 & 6.0 & 6.0 & 6.0 \\
Mezcla de vitaminas y minerales** & 3.0 & 3.0 & 3.0 & 3.0 \\
Vitamina C** & 0.4 & 0.4 & 0.4 & 0.4 \\
Benzoato de Sodio & 0.2 & 0.2 & 0.2 & 0.2 \\
Cloruro de colina & 0.1 & 0.1 & 0.1 & 0.1 \\
$\alpha$-Tocopherol & 0.01 & 0.01 & 0.01 & 0.01 \\
\hline Composición proximal (\% base en materia seca) & & & \\
\hline Proteína cruda (\%) & 30.2 & 30.5 & 40.1 & 40.3 \\
Tola de lipidos (\%) & 8.2 & 16.1 & 8.3 & 16.2 \\
Cenizas (\%) & 13.5 & 13.5 & 16.8 & 17.0 \\
Extracto libre de nitrógeno*** & 48.0 & 39.9 & 34.8 & 26.5 \\
Coeficiente de digestibilidad $(\%)$ & $79.9 \pm 2.0 \mathrm{a}$ & $65.8 \pm 4.9 \mathrm{~b}$ & $46.9 \pm 3.5 \mathrm{c}$ & $44.5 \pm 4.1 \mathrm{c}$ \\
\hline
\end{tabular}

Los valores se expresan como media y $( \pm) \mathrm{SD},(\mathbf{n}=3)$, los valores dentro de la misma fila con diferentes letras sobrescritas significan diferencias significativas $(p<0.05)$.

equivalente al $8 \%$ de su biomasa inicial. Cada $15 \mathrm{~d}$ los peces se pesaron en $(\mathrm{g})$ y medieron en $\mathrm{mm}$. Los reservorios se limpiaron todos los días y se registró la supervivencia durante los $60 \mathrm{~d}$ del experimento. Los peces se alimentaron a las 9:00, 14:00 y 18:00 todos los días hasta saciedad.

Para evaluar el crecimiento se calculó la ganancia en peso (GP\%):

$$
G P(\%)=\left(\frac{(\text { peso final }- \text { peso inicial })}{\text { peso inicial }}\right) \times 100
$$

Tasa especifica de crecimiento (TEC):

$T E C=((\ln ($ peso final $)-\ln ($ peso inicial $)) T$ iempo en dias $) x 100$

El Coeficiente termal de crecimiento (CTC) se calculó con la formula:

$$
C T C=\frac{\sqrt[3]{\text { peso final }}-\sqrt[3]{\text { peso inicial }}}{T^{\circ} \mathrm{C} x \text { dias }} \times 1000
$$

donde: $\mathrm{T}^{\circ} \mathrm{C}=29$ y $\mathrm{D}$ días $=60$.

Se realizó el análisis proximal de las cuatro dietas experimentales y de la zona dorsal (músculo) de los organismos al inicio y al final del experimento. Para los análisis proximales se capturaron tres peces por unidad experimental de forma aleatoria y se sacrificaron mediante un shock térmico (descenso de temperatura con agua y hielo ) para evitar el estrés (Diaz-Villanueva y Robotham 2015). Todos los análisis se realizaron por triplicado.

La determinación de humedad, proteína total, grasa total y cenizas de los alimentos y del músculo se realizaron siguiendo los protocolos de la AOAC (1995). Los músculos de tres peces de cada unidad experimental se mezclaron para los análisis. La determinación de humedad se realizó por diferencia gravimétrica, pesando $1.0 \mathrm{~g}$ de muestra, para luego secarlo en una estufa a $100{ }^{\circ} \mathrm{C}$, hasta peso constante. La determinación del contenido de proteína cruda se realizó con el método de micro Kjeldahl y se multiplico por el factor de 6.25. El contenido de lípidos se determinó con el método de extracción de Soxhlet con la ayuda de hexano como solución extractora. Las cenizas se determinaron por calcinación de una muestra en una mufla a 550 ${ }^{\circ} \mathrm{C}$ por $6 \mathrm{~h}$. La determinación del extracto libre de nitrógeno se calculó por la diferencia de la materia seca con la formula ELN = 100 - (\% proteína cruda $+\%$ lípidos totales $+\%$ de cenizas.

Para determinar la digestibilidad de las dietas, se colectaron heces de las unidades experimentales por $10 \mathrm{~d}$, con ayuda de un colector de heces. Una vez colectadas las heces se mantuvieron en 


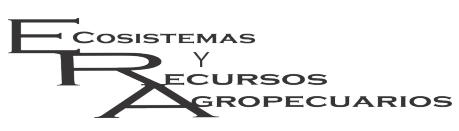

recipientes plásticos en congelación a $-20{ }^{\circ} \mathrm{C}$, para su posterior análisis. La determinación del coeficiente de digestibilidad aparente (CDA) de los nutrientes del alimento, se realizó con la determinación de cenizas insolubles en ácido del alimento y de las heces, con el método propuesto por Tejada-de Hernández (1992) modificado por Montaño-Vargas et al. (2002).

Las cenizas insolubles en ácidos $(\mathrm{CIA})$ se determinaron con la siguiente fórmula.

$$
C I A(\%)=\left(\frac{\text { Peso ceniza, } g-\text { Peso del crisol, },}{\text { Muestra seca,g }}\right) \times 100
$$

La digestibilidad aparente (DA) se determinó con la fórmula.

$$
D A(\%)=100-\left(\frac{100 \times \% \text { cenizas en el alimento }}{\% \text { de cenizas en las heces }}\right)
$$

Para determinar las diferencias estadísticas entre los tratamientos, se realizó una prueba de homogeneidad entre las repeticiones de los datos y después se realizó un análisis de varianza de una vía (ANOVA). El nivel de significación se estableció con una $p<0.05$. Cuando se detectaron diferencias estadísticas, se utilizó una prueba post hoc de Tukey para identificar las diferencias estadísticas entre los tratamientos. Los análisis se realizaron con el programa estadístico Statistica versión 6.1.

\section{RESULTADOS Y DISCUSIÓN}

No se encontraron diferencias estadísticas para la ganancia de peso de juveniles de $D$. latifrons en los distintos niveles de proteína y lípidos en la dieta (Tabla 2). Lo indica que con $30 \%$ de proteína y $8 \%$ de lípidos es suficiente para producir un adecuado crecimiento, lo que demuestra que los peces alimentados con dietas bajas en proteínas utilizaron con mayor eficiencia la proteína dietética, en comparación con aquellos alimentados con dietas altas de proteínas y lípidos. Estos resultados permiten el diseño de dietas para esta especie con menores niveles de inclusión de insumos con alto precio en el mercado, en particular la proteína, o el uso de alimentos comerciales de menor costo. En la nutri-
Requerimiento proteína-lípidos de Dormitator latifrons

Ecosist. Recur. Agropec.

5(14):345-351,2018

ción acuícola la alimentación es uno de los factores de mayor atención, debido a que llega a representar más del $50 \%$ de los costos de producción, debido a la falta de insumos y el encarecimiento de los ingredientes utilizados (Tacon et al. 2011, NRC 2011).

Se sabe que el alimento tiene efecto en el crecimiento de los peces, pero depende de la especie, habitos alimenticios, talla, edad, condición fiiologica y de las condiciones físicas y quimicas del agua de mantenimiento (Phillips et al. 1998). Pero para $D$. latifrons no hay estudios publicados sobre nutrición que permitan ser utilizados para establecer condiciones básicas para su mantenimiento en confinamiento, ni estudios sobre su fisiología. Al respecto Yañez-Arencibia y Díaz González (1977) mencionan que esta especie es consumidora de detritos y restos de plantas. Mientras que CastroRivera et al. (2005) abordan la conversión alimenticia por medio del crecimiento de cultivos puros de machos, hembras y mixtos, con alimentos comerciales con porcentajes de proteían y lípidos de 30 y $5 \%$, respectivamente.

Estudios en juveniles de tilapia roja híbrida (Oreochromis mossambicus y $O$. niloticus) alimentados con 15, 20 y $30 \%$ de proteína y $6,12,18$, $24 \%$ de lípidos, indican que los mejores resultados de crecimiento lo tuvieron las dietas que contenían la mayor inclusión de lípidos (18\%), sin importar el nivel de proteína (De Silva et al. 1991). Mientras que para Carassius auratus (Cyprinidae) se reporta un mejor crecimiento con dietas que contenían 40 y $45 \%$ de proteína y $8 \%$ lípidos, mientras que dietas con menor cantidad de proteína y lípidos tuvieron un bajo crecimiento (Castillo et al. 2014). Para especies carnívoras marinas se reporta que juveniles de Totoaba (Totoaba macdonaldi) se tuvo el mejor crecimiento con la dieta que contenía $52 \%$ de proteína y $8 \%$ de lípidos (Rueda-López et al. 2011). Para la corvina Golfina (Cynoscion othonopterus) se encontró que el mejor tratamiento fue con la dieta que contenía $40 \%$ de proteína y $8 \%$ de lípidos (Pérez-Velázquez et al. 2015). Los resultados demuestran que no es posible adaptar a cualquier especie las dietas diseñadas para una especie en particular, debido a que los requerimien- 
Tabla 2. Índices biológicos de juveniles de $D$. latifrons alimentados con cuatro dietas experimentales con diferentes niveles de proteínas y lípidos.

\begin{tabular}{lcccc}
\hline Índices Biológicos & $30-8$ & $30-16$ & $40-8$ & $40-16$ \\
\hline Peso inicial (g) & $17.3 \pm 0.3$ & $17.2 \pm 0.3$ & $17.3 \pm 0.5$ & $17.7 \pm 0.1$ \\
Peso final (g) & $73.6 \pm 5.2$ & $71.2 \pm 6.9$ & $75.6 \pm 8.5$ & $62.2 \pm 7.5$ \\
Longitud inicial (cm) & $10.8 \pm 1.7$ & $10.8 \pm 1.7$ & $10.8 \pm 1.7$ & $10.8 \pm 1.7$ \\
Longitud final (cm) & $17.2 \pm 2.5$ & $15.8 \pm 0.4$ & $16.2 \pm 0.2$ & $14.7 \pm 1.2$ \\
Ganancia total en peso (g) & $56.2 \pm 5.5$ & $54 \pm 6.7$ & $58.3 \pm 8.7$ & $44.4 \pm 7.5$ \\
Peso ganado (\%) & $425.1 \pm 37.6$ & $414 \pm 36.7$ & $438.5 \pm 55.5$ & $351.9 \pm 43.8$ \\
Peso ganado por día (g/día) & $1.2 \pm 0.1$ & $1.1 \pm 0.1$ & $1.2 \pm 0.1$ & $1.0 \pm 0.1$ \\
Tasa especifica de crecimiento & $2.41 \pm 0.1$ & $2.36 \pm 0.1$ & $2.46 \pm 0.2$ & $2.09 \pm 0.2$ \\
Coeficiente termal de crecimiento & $0.91 \pm 0.0$ & $0.89 \pm 0.0$ & $0.93 \pm 0.0$ & $0.78 \pm 0.0$ \\
Supervivencia (\%) & $93.3 \pm 5.7$ & $93.3 \pm 5.7$ & $100 \pm 0.0$ & $86.6 \pm 23$ \\
\hline Los índices biológicos se calcularon a partir de 60 d de experimentación. &
\end{tabular}

Tabla 3. Análisis proximal de tejido muscular de juveniles de $D$. latifrons alimentados con cuatro dietas experimentales que contienen diferentes niveles de proteínas y lípidos.

\begin{tabular}{lccccc}
\hline Índices Biológicos & \multicolumn{5}{c}{ Tratamientos experimentales } \\
\hline & Inicial & $30-8$ & $30-16$ & $40-8$ & $40-16$ \\
& Composición proximal de tejido muscular (\% materia seca) \\
Proteína cruda (\%) & $78.2 \pm 10.6$ & $81.8 \pm 3.2$ & $76.7 \pm 4.6$ & $78.8 \pm 2.3$ & $78.3 \pm 3.5$ \\
Lípidos (\%) & $4.3 \pm 0.7$ & $5.3 \pm 0.8$ & $5.9 \pm 0.6$ & $5.7 \pm 1.1$ & $6.1 \pm 1.0$ \\
Cenizas (\%) & $6.0 \pm 0.7$ & $7.5 \pm 1.5^{b}$ & $8.8 \pm 0.8^{a}$ & $9.1 \pm 0.9^{a}$ & $7.9 \pm 0.5^{a b}$ \\
Extracto libre de nitrógeno* & 11.5 & 5.4 & 8.6 & 6.3 & 7.7 \\
\hline Los valores se dan como media y $( \pm) \mathrm{SD}, \mathrm{n}=3$, los valores dentro de la misma fila con diferentes letras \\
significan diferencias significativas $(p<0.05)$, & * Extracto libre de nitrógeno = 100 - (\% de proteína \\
cruda + lípido total + \% de ceniza).
\end{tabular}

tos de los nutrientes pueden diferir de manera importante. Las altas concentraciones de proteínas y lípidos en una dieta, no necesariamente resultan en mayor crecimiento y estado de salud de los peces, ya que se ha demostrado que en algunos casos los altos niveles de proteína y lípidos pueden limitar la regeneración múscular (Rueda-López et al. 2011), piel, células sanguíneas y enzimas, lo que ocasiona obesidad o infiltración grasa del hígado (Wang et al. 2013). Lo que incrementa la susceptibilidad a enfermedades, pérdida de pigmentación y erosión en las aletas (Riaño-Castillo et al. 2014).

La composición proximal del músculo inicial y final, después de $60 \mathrm{~d}$, fue similar en los cuatro tratamientos (Tabla 3), lo que indica que a pesar de las mayores concentraciones de nutrientes, estos no llegan a metabolizarse para favorecer el desarrollo tisular, lo que prueba que hay un rango óptimo para la utilización de proteínas y lípidos. La digestibilidad aparente tuvo diferencias estadísticas con valores del 44.4 al $77.9 \%$, presentando la mayor digestibilidad la dieta con 30 y $8 \%$ de proteína y lípidos, respectivamente (Tabla 1). Al respecto Pezzato et al.
(2002), mencionan que variaciones en la composición lipídica de la dieta, pueden originar diferencias en la digestibilidad.

En el periodo de cuarentena se observo que los peces tuvieron una rápida adaptación al alimento comercial, consumiendo alimento extruido flotante y peletizado. Con relación a los parámetros fisicoquímicos del agua (Tabla 4), se registraron valores de temperatura que concuerdan con las temperaturas preferentes de la especie, de entre 21 y $30{ }^{\circ} \mathrm{C}$ (FAO 2010). D. latifrons, no mostró diferencias estadísticas en crecimiento, ni supervivencia con dietas que contiene inclusiones del $30 \mathrm{y}$ $40 \%$ de proteína y del 8 al $16 \%$ de grasa. De acuerdo con los resultados obtenidos una dieta con $30 \%$ de proteína y $8 \%$ de lípidos puede ser suficiente para promover un adecuado crecimiento. Estos resultados son los primeros obtenidos para esta especie con interés potencial para su cultivo. 
Tabla 4. Valores promedio y desviación estándar de los parámetros físicoquímicos del agua del cultivo de $D$. latifrons durante $60 \mathrm{~d}$ en un sistema de recirculación.

\begin{tabular}{lc}
\hline Parametros & \\
\hline Salinidad (UPS) & $1.0 \pm 0.2$ \\
Temperatura $\left({ }^{\circ} \mathrm{C}\right)$ & $29.2 \pm 1.0$ \\
$\mathrm{pH}$ & $7.7 \pm 0.4$ \\
Oxígeno $\left(\mathrm{mg} / \mathrm{L}^{-1}\right)$ & $5.4 \pm 0.5$ \\
Amonio $\left(\mathrm{mg} / \mathrm{L}^{-1}\right)$ & $0.06 \pm 0.01$ \\
Nitritos $\left(\mathrm{mg} / \mathrm{L}^{-1}\right)$ & $2.2 \pm 0.4$ \\
Nitratos $\left(\mathrm{mg} / \mathrm{L}^{-1}\right)$ & $0.52 \pm 7.3$ \\
\hline
\end{tabular}

\section{LITERATURA CITADA}

AOAC (1995) Official Methods of Analysis 16 th edn. Association of Official Analytical Chemists. Arlington, VA, USA. 684p.

Castillo RE, Torres MA, Hurtado GH, Gómez RE (2014) Efecto del nivel de proteína cruda en la dieta sobre el crecimiento y excreción de nitrógeno amoniacal de Carassius auratus (Cyprinidae) Linnaeus (1758) bajo condiciones de laboratorio. Revista Universidad Militar de Nueva Granada 11: 1 34-37.

Castro-Rivera R, Aguilar-Benítez G, Hernández-Girón J (2005) Conversión alimenticia en engordas puras y mixtas de Popoyote (Dormitator latifrons Richardson) en estanques de cemento. AquaTIC 23: 45-52.

De Silva SS, Gunasekera RM, Shim KF (1991) Interactions of varying dietary protein and lipid levels in young red tilapia: Evidence of protein sparing. Aquaculture 95: 305-318.

Diaz-Villanueva J, Robotham H (2015) Comparacion de dos métodos de sacrificio en truchas arcoíris (Oncorhynchus mykiss). Latin American Journal of Aquatic Research 43: 287-294

FAO (2010) Peces nativos de agua dulce de América del Sur de interés para la acuicultura: Una síntesis del estado de desarrollo tecnológico de su cultivo. Serie Acuicultura en Latinoamérica. Numero 1. Organización de las Naciones Unidas para la Agricultura y la Alimentacion Roma, Italia. 200p.

FAO (2016) El estado mundial de la pesca y la acuicultura 2016. Contribución a la seguridad alimentaria y la nutrición para todos. Organización de las Naciones Unidas para la Agricultura y la Alimentacion. Roma, Italia. 224p.

Freire-Lascano CA (2013) Experiencias en el manejo del Chame (Dormitator latifrons) en la Cuenca del Río Guayas, Ecuador. Revista Electrónica de Ingeniería en Producción Acuícola 7: 1-13.

González-Félix ML, Minjarez-Osorio C, Pérez-Velázquez M, Suárez-Jiménez GM, Ibarra-Garcíaparra GE (2014) The Cortez flounder Paralichthys aestuarius as a candidate species for aquaculture: First report on growth in captivity in response to varying dietary protein levels. Aquaculture 420-421: 225-230.

Larumbe E (2002) Algunos aspectos biológicos de los popoyotes (Dormitator latifrons) en cautiverio. Revista Panorama Acuícola 24-25. http://fis.com/panoramacuicola/noticias/noticia\%203.htm. Fecha de consulta 12 de junio de 2017

López-López VV, Rodríguez-Montes de Oca GA, Galavíz MA, Román-Reyes C, Medina-Hernández EA, Dabrowski K, et al. (2015) Descripción histológica comparativa del desarrollo del sistema digestivo y visual de larvas de chame Dormitator latifrons (Pisces: Eleotridae). Latin American Journal of Aquatic Research 43: 484-494. 
Montaño-Vargas J, Shimada A, Vásquez C, Viana MT (2002) Methods of measuring feed digestibility in the green abalone (Haliotis fulgens). Aquaculture 213: 339-346.

NRC (2011) Nutrient requirements of fish and shrimp. National Research Council. The National Academies Press. Washington, USA. 392p.

Okolodkov YB, Bastida-Zavala R, Ibáñez LA, Chapman WJ, Suarez-Morales E, Pedroche F, et al. (2007) Especies acuáticas no indígenas en México. Ciencia y Mar 32: 29-67.

Pérez-Velázquez M, González-Félix ML, Viana MT, Lazo-Corvera JP, Maldonado-Othón A (2015) Effects of dietary protein and lipid levels on growth and body composition of the Gulf corvina, Cynoscion othonopterus. International Journal of Aquatic Science 6:11-28.

Pezzato LE, Miranda EC, Barros MM, Pinto LGQ, Furuya WM et al. (2002) Digestibilidade aparente de Ingredientes pela Tilápia do Nilo (Oreochromis niloticus). Revista Brasileña de Zootecnia 31: 1595-1604

Phillips AT, Sumerface CR, Clayton AR (1998) Feeding frequency effects on water quality and growth of Walleye fingerlings in intensive culture. The Progressive Fish Culturist 60: 1-8

Riaño-Castillo RE, Torres-Mesa MA, Hurtado-Giraldo GH, Gómez-Ramírez E (2014) Efecto del nivel de proteína cruda en la dieta sobre el crecimiento y excreción de nitrógeno amoniacal total de Carassius auratus (Cyprinidae) Linnaeus (1758) bajo condiciones de laboratorio. Revista Facultad de Ciencias Básicas 11: 34-47.

Rueda-López S, Lazo JP, Correa-Reyes G, Viana MT (2011) Effect of dietary protein and energy levels on growth, survival and body composition of juvenile Totoaba macdonaldi. Aquaculture 319: 385-390.

Tacon A, Hasan MR, Metian M (2011) Demand and supply of feed ingredients for farmed fish and crustaceans: Trends and prospects. FAO, Fisheries and aquaculture technical paper No. 564. Rome, Italy. 87p.

Tejada-de Hernández I (1992) Control de calidad y análisis de alimentos para animales. Sistema de educación continua en producción animal. México. 397p.

Vega-Villasante F, Cueto-Cortes L, Basto-Rosales ME, Badillo-Zapata D, Chong-Carrillo O, et al. (2017) Ocurrence of Argulus sp. in Dormitator latifrons culture: prevalence, mortality and treatment. Revista Bio Ciencias 4. Article ID 04.06.05. Doi: 10.15741/revbio.04.06.05.

Yañez-Arancibia A, Díaz-González G (1977) Ecología trofodinámica de Dormitator latifrons (Richardson) en nueve lagunas costeras del Pacífico de México (Pisces: Eleotridae). Anales del Centro de Ciencias del Mar y Limmología 4: 125-140

Wang YYMa, Shi GJ, Liu Y, Guo DS, Yang JX, Chen YH (2013) Effects of dietary protein and lipid levels on growth, feed utilization and body composition in Pseudobagrus ussuriensis. Aquaculture Nutrition 19: 390.398. 
